\title{
Integration of numerical analysis, virtual simulation and finite element analysis for optimum design of worm gearing
}

\author{
Daizhong $\mathrm{Su}^{\mathrm{a}}$ and Datong Qin ${ }^{\mathrm{b}}$ \\ ${ }^{a}$ Corresponding author, School of Engineering, The Nottingham Trent University, \\ Nottingham, NG1 4BU, UK; http://domme.ntu.ac.uk/mechdes \\ ${ }^{b}$ State Key Laboratory of Mechanical Transmission, Chongqing University, Chongqing, P R China
}

\begin{abstract}
Due to the complicated tooth geometry of worm gears, their design to achieve high capacity and favourable performance is a tedious task, and the optimum design is hardly to obtain. In order to overcome this problem, the authors developed an integrated approach which consists of three modules: numerical analysis, three-dimensional simulation and finite element analysis. It provides a powerful tool for optimum design of worm gears and is a general approach applicable for various types of worm gears. As case studies, two designs using the approach have been conducted, one for cylindrical involute worm gearing and the other for double enveloping worm gearing.
\end{abstract}

Keywords: worm gears, numerical analysis, virtual manufacturing, finite element analysis, CAD/CAM/CAE

\section{Introduction}

Worm gears are essential mechanical components, with wide applications ranging from toys to heavy industrial machinery. They have several advantages over other types of drives, such as high speed ratio, smooth transmission, low level of noise and compact size.

However, due to the complexity of their tooth geometry, the design of worm gear drives to achieve high capacity and favourable performance is a tedious task, and the optimum design is hardly to obtain. In order to overcome this problem, the authors developed a comprehensive computer aided approach for optimum design of worm gears, which integrate multiple techniques including numerical analysis, advanced $\mathrm{CAD}$, virtual simulation, and finite element analysis. It is a general approach applicable for various types of worm gears.

In this paper, the overview of the integrated approach is given first, together with a review of recent development in related areas; then, application of the approach into two types of worm gears, involute worm gearing and double enveloping hourglass worm gearing, is presented, which proves that the approach is a powerful tool for worm gear design.

\section{The integrated approach}

As shown in figure 1, the approach includes three modules:

Module 1 -- numerical analysis. It comprises of set of mathematical models derived based on differential geometry theory to calculate the complicated tooth geometry.

Module 2 -- three dimensional simulation. In this module, 3D models of worm and wheel are created within the advanced CAD package Pro/Engineer. There are two methods to create the models: (i) using the numerical data of the tooth surfaces obtained from numerical analysis module, or (ii) both the worm and wheel are virtually produced in Pro/Engineer by simulating the manufacturing process. Then the worm and the wheel are assembled together to form the worm-wheel assembly model. Using the assembly model, the tooth contact pattern can be simulated. With investigation of the simulation results, the design parameters of the worm drive can be modified in order to achieve a better contact pattern.

Module 3 -- finite element analysis. Based on the worm and wheel models created in module 2, this module can calculate the mesh stiffness, tooth bending and contact stresses. Based on the results obtained, the load distribution, transmission errors and other meshing characteristics can be analysed as well.

In comparison with existing research, this new approach has the following features:

Controllable localised tooth contact with less sensitivities to errors. In order to reduce sensitivities to errors arising from manufacturing and assembly, the concept localised tooth contact has been introduce. However, the existing methods may achieve localised contact, but cannot ensure the contact area is placed at an ideal position and hence the mesh quality is uncontrollable.

With this approach, the worm and wheel are initially designed in Module 1. Module 2 provides a 'live' environment for the designer to visually investigate the tooth contact of the designed worm-wheel pair and change the relative positions of the worm and wheel to place the localised contact at an ideal position on the tooth surface. The design parameters related to the new relationship are then feed back to Module 1 for further calculation. In this way, the localised tooth contact is controllable.

Loaded tooth contact analysis (LTCA). With the LTCA, the tooth deformation, stresses and load distribution can be estimated more accurately. Although considerable work has been done for other types of gears, for example, [5,6], LTCA for worm gears has not been seen. In the finite element analysis module of this 
approach, the finite element modelling, load share analysis and transmission errors estimation are based on the LTCA, which provide more accurate results than existing methods.

Virtual manufacturing. The worm and wheel tooth surfaces are generated based on parametric modelling. The generation procedure is a virtual manufacturing process. The threedimensional simulation module not only simulates the contact pattern on the tooth surface, but also detects the tooth interference that may occur during tooth engagement.

Integration of multiple computer aided techniques. This research integrates numerical analysis, advanced $\mathrm{CAD}$, virtual manufacturing and finite element analysis into a single approach. These multiple techniques complement with each other to form a powerful tool for worm gear design. Within the approach, the advantages of each technique have been best utilised and their drawbacks have been avoided.

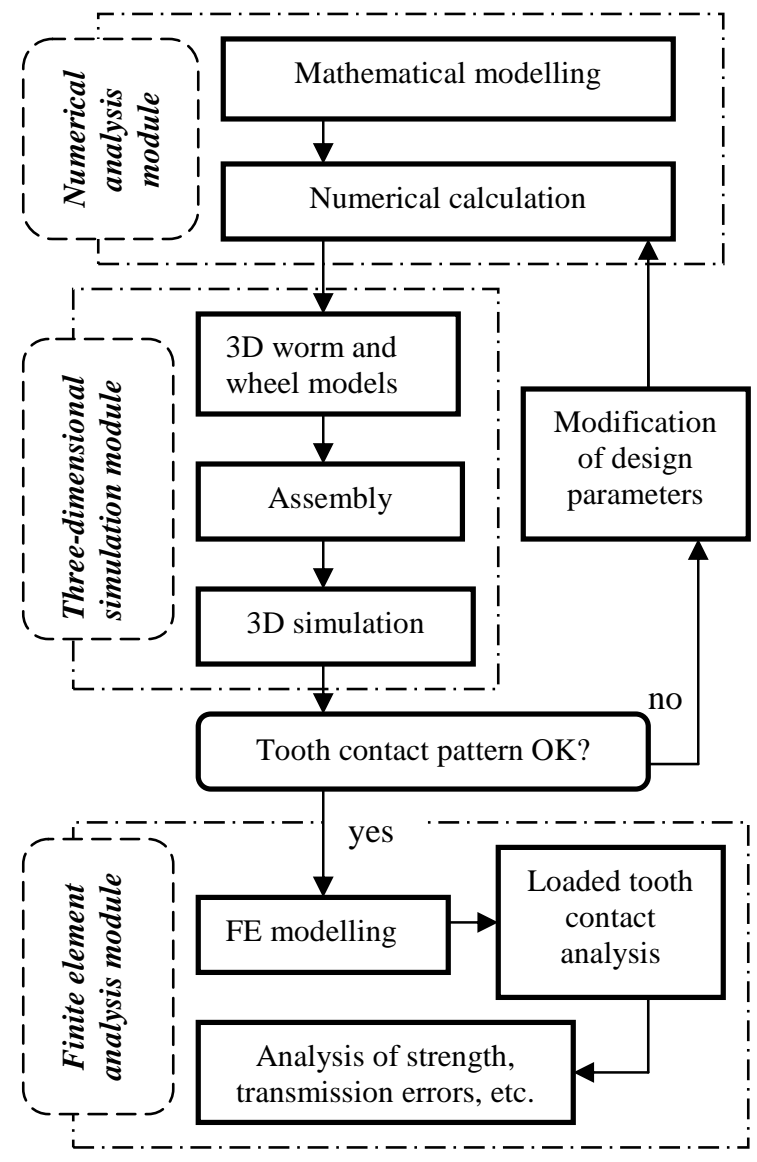

Figure 1 Flowchart of the approach

3. Application of the integrated approach into optimum design of involute worm gearing with localised tooth contact

\subsection{Mathematical models for modifying the tooth geometry}

The procedure of the tooth geometrical modification includes the following steps (for further details, see [3]):

Step 1 - Determination of the reference for tooth geometry modification. To analyse the tooth modification, it is necessary to define a calculating reference. The position of the reference, its relative velocity and normal are determined based on gearing meshing theory [4].

Step 2 - Modification of structural parameters of worm gearing. The worm diameter and its lead angle are changed to achieve optimum worm tooth profile and the hob-mounting angle $\theta_{m}$ is altered to adjust the wheel tooth surface. With the optimum combination of the structure parameters, an ideal contact pattern can be achieved. In order to ensure the continuous meshing process, the modified worm must have the same normal pitch at the reference point as the original one.

Step 3 - Iterative calculation for parameter optimisation. The optimum combination of the structure parameters is achieved through an iterative calculation process. Obviously, the modified worm gear drives should still satisfy the meshing equation. Thus, the iteration process is based on the meshing equation. The constraint condition consists of two assumptions: equal normal of the modified worm and wheel at the reference point, and equal normal pitch between the modified worm and the hob.

Step 4 - Generate the modified tooth surfaces. With the results obtained from the iteration process, the modified worm tooth surface can be generated. The involute worm gearing is used here as an example. The modified worm tooth profile is represented as below:

$\vec{r}_{1}=\left(\begin{array}{l}x_{1} \\ y_{1} \\ z_{1}\end{array}\right)=\left(\begin{array}{l}r^{*} \cdot \cos \phi_{u}+u \cdot \cos \delta^{*} \cdot \sin \phi_{u} \\ r^{*} \cdot \sin \phi_{u}-u \cdot \cos \delta^{*} \cdot \cos \phi_{u} \\ -u \cdot \sin \delta^{*}+p \phi_{u}\end{array}\right)$

where, $r^{*}$ is the radius of base cylinder of the worm, $\delta^{*}$ is the lead angle of the worm, and $p$ is the pitch of the worm.

The modification of the wheel tooth surface of the wheel is based on changing the hob-mounting angle $\theta_{h}$. The modification enables the tooth surface to be in internal tangency meshing with the worm, and places the local contact area in an appropriate position. The tooth surface with modified $\theta_{h}$ is expressed as

$\vec{r}^{(2)}=\vec{r}^{(2)}\left(u, \phi_{u}, \phi_{1}, \theta_{h}\right)=M_{2 \theta}\left(\phi_{2}\left(\phi_{1}\right)\right) \cdot M_{\theta 1}\left(\phi_{1}, \theta_{h}\right) \cdot \vec{r}^{(1)}$

$f\left(u, \phi_{u}, \phi_{1}, \theta_{h}\right)=0$

where $f=0$ is the worm-wheel meshing equation; $M_{2 \theta}, M_{\theta 1}$ are the transfer matrices from $\Sigma_{\theta}$ to $\Sigma_{2}$ and from $\Sigma_{1}$ to $\Sigma_{\theta}$ respectively; $\theta_{h}$ is the hob-mounting angle.

\subsection{Three-dimensional modeling and virtual manufacturing}

Two methods have been developed to establish the 3D models of worm, wheel and their assembly: a method based on numerical analysis results (NAR) and a method based on the cutting process applied in manufacturing practice (CPM).

The NAR method produces accurate tooth geometry and provides useful formulae for complicated calculation such as induced normal curvatures, relative tooth velocities, etc. However, it is very complicated and the mathematical models of tooth geometry must be available. For further details, see [1].

The CPM method does not require the mathematical models of the worm and wheel tooth surfaces. Using the CPM method, the worm gear is produced by virtual cutting process in $\mathrm{CAE}$ package Pro/Engineer. The 3D modelling is based on the cutting process applied in manufacturing practice. The advantages of 
using this method are that the 3D worm gear model is parametric and very flexible to change. The worm gear drive set can be assembled and virtually run in the computer to check the interference or to see the localised tooth contact. Therefore, it is a valuable visualised aid for gear researchers and engineers to optimal design of modified worm gears.

The procedure of 3D modelling and simulation is as follows:

i. Prepare worm, hob and wheel design parameters

ii. Calculate and construct the through axis cross-section profile

iii. Cut the worm by helical sweep cut

iv. Cut the wheel by patterning helical sweep cut

v. Assemble worm gear drive set

vi. Virtually run the worm gear drive in the computer to check the contact path and interference between worm and worm gear teeth

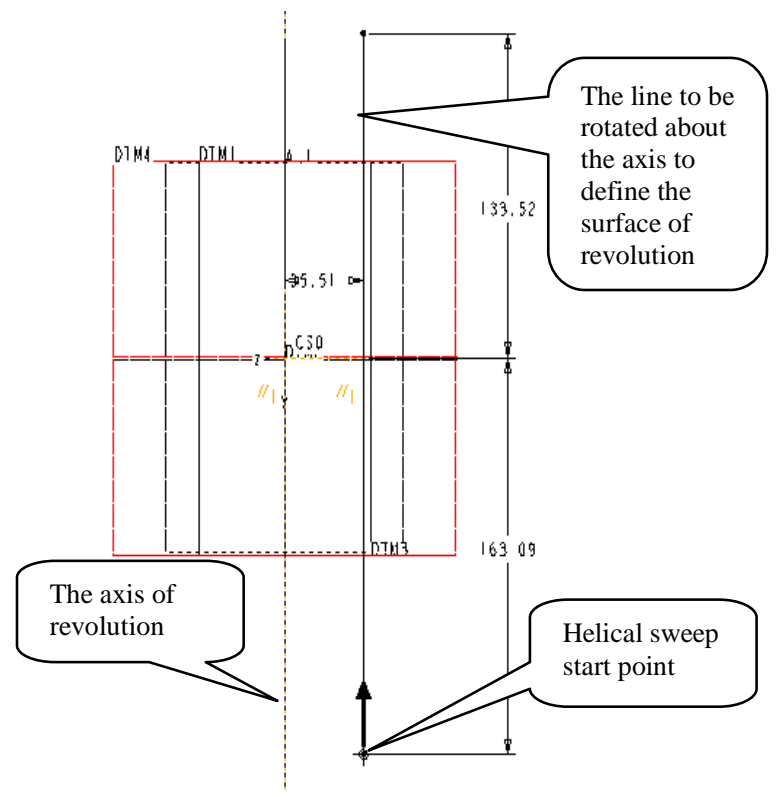

Figure 2. Helical sweep profile

Worm cut. A worm cylinder base with its outside diameter identical to the worm outside diameter is produced in Pro/Engineer first. The advanced constructing feature option, Helical Sweep Cut, is used to simulate the worm tooth cutting process. In order to achieve this, the tooth cutter profile is needed. For ZA worm, the hob blade is a straight line in the through axis plane, therefore the Through Axis option in helical sweep cut is used. For ZN worm, the hob blade is normal to the helical curve so the Normal To Trajectory Helical Sweep Cut option is used. For ZI worm, its cross section can be obtained from the surface equations (1). After the through axial cross section of worm tooth profile is produced, the helical sweep profile is determined as shown in Figure 2.

Wheel hobbing. The features of the wheel are much more difficult to produce than those of the worm. The wheel teeth conjugate with worm teeth and the surface profile is dependent on worm. In practical wheel cutting, except the clearance, the worm hob is identical to the worm. The hobbing process is the same as the actual worm gear drive operation. So the produced wheel can be conjugated with the worm.

Figure 3 shows the mounting relation of the worm hob axis and the wheel base. The worm cutter and wheel axes are perpendicular to each other. The wheel base and its hob rotate around their own axes at a constant ratio.

For further details of the worm and wheel virtual manufacture, see [2].

Simulation and analysis of the tooth contact are conducted with the 3-D simulation module. It presents a virtual meshing process for the worm gear drive. Using the assembly model shown in Figure 4, the 3-D simulation of localized tooth contact can be conducted. The tooth contact pattern and the moving direction of the localized contact area can be predicted; moreover, the interference between the modified tooth surfaces can be detected, and, hence, it can be avoided at design stage. An example of the simulation results obtained is shown in Figure 5.

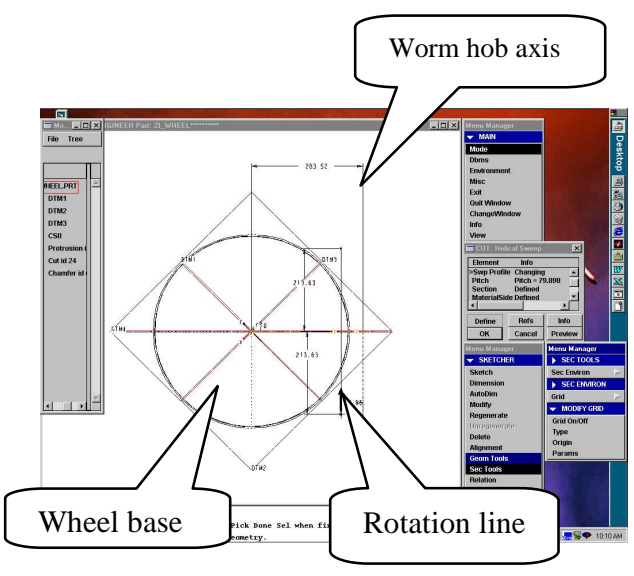

Figure 3 Worm gear hob mounting relations

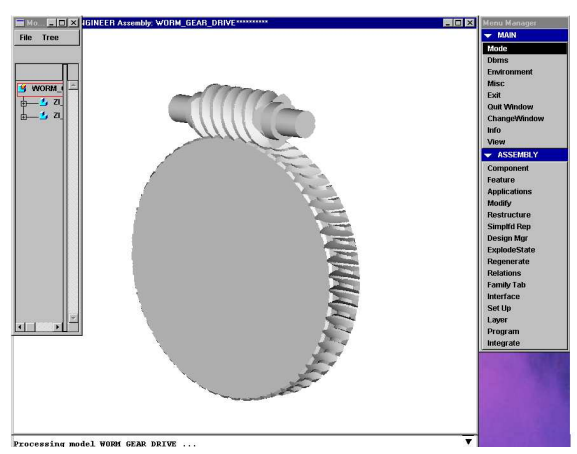

Figure 4. The assembled worm gear drive set

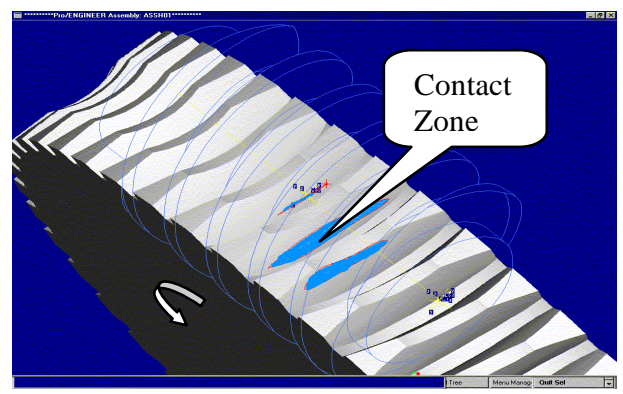

Figure 5 An example of 3-D simulation of tooth contact 


\subsection{Finite element analysis}

After the user satisfied the 3D simulation results, the 3-D models created in Pro/Engineer are exported as an IGES file which is then imported into the software ANSYS 5.3. When the IGES file is retrieved in ANSYS, it is usually only a geometric model with the surface information. Within the pre-processor of ANSYS, it is necessary to merge the surfaces to create proper volumes. After that, the proper element type and material property need to be chosen and the mesh of models is then performed.

FE model of wheel. Due to the complexity of the tooth surface geometry, it is difficult to apply mapped meshing for the wheel model. In order to resolve this problem, it is necessary to divide the body into some individual units of 6-sided volumes so that the software can implement the mapped mesh.

FE model of worm. To create a whole model of the worm, a useful approach for finite element modelling of helical geometry has been developed. The idea to develop a complete worm FE mesh is based on the fact that mesh extrusion of shell elements can generate $3 \mathrm{D}$ elements and this function can produce elements to fit helical shapes such as worm profiles. A function command called VEXT operation in ANSYS5.3 is applied to perform the mesh extrusion. Because a rotation of the shell elements around an axis is allowed during the VEXT operation, the extrusion process is able to produce a helical shape mesh.

Using the finite element models developed, the loaded tooth contact, tooth stiffness, load distribution, bending and contact stresses and transmission errors can be analyzed, for further details, see $[8,12]$.

\section{Application of the integrated approach into optimum design of double enveloping worm gearing}

A sketch of a double enveloping hourglass worm gear pair is shown in Figure 6. Due to the shape of the worm and the generation nature of the tooth surfaces, it has more tooth pairs in mesh than cylindrical worm gears for similar transmission parameters, and there are two contact lines on one tooth surface. Therefore, this type of worm gears has higher loading capacities than other types of worm gears.

The integrated approach has also been applied into this types of worm gears: the numerical analysis module has been completed and reported in [7]; the three-dimensional module is currently in the process of development as part of a collaborative project between The Nottingham Trent University, UK and the State Key Laboratory of Mechanical Transmission, China; and finite element analysis module is presented in this section.

\subsection{Gear geometry and errors}

As shown in Figure 6, in the machining of double enveloping hourglass worm gears, a plane-grinding wheel which is tangent to a generating circle generates the worm. The generating circle and the worm axis are located in the same horizontal plane. The plane-grinding wheel is attached to the rotary table of hob machine and inclines with an angle $\beta$ to the axis of the rotary table. The worm blank pivots around its axis with angular velocity $\omega_{1}$, meanwhile the tool plane pivots around the axis of the rotary table with angular velocity $\omega_{2}$, and the worm surface is thus generated. When the worm is substituted by a hob with same theoretical profile as the worm and a blank of the worm wheel is placed on the rotary table, with the same setting and motion relations as that in worm machining, then the wheel is produced. For numerical calculation of the tooth surfaces and related geometry, see [7].

In the manufacturing of worm gears, machining and assembling errors are inevitable. In this research, the assembling errors in centre distance of the worm gearing, in axial direction of the worm, in axial direction of the wheel, and the error of the crossing angle of the worm and wheel axes are introduced to investigate the influences of manufacturing errors on the tooth contact. These errors are denoted as $\Delta \mathrm{A}, \Delta \mathrm{B}, \Delta \mathrm{C}$ and $\Delta \gamma$, as shown in Figure 6.
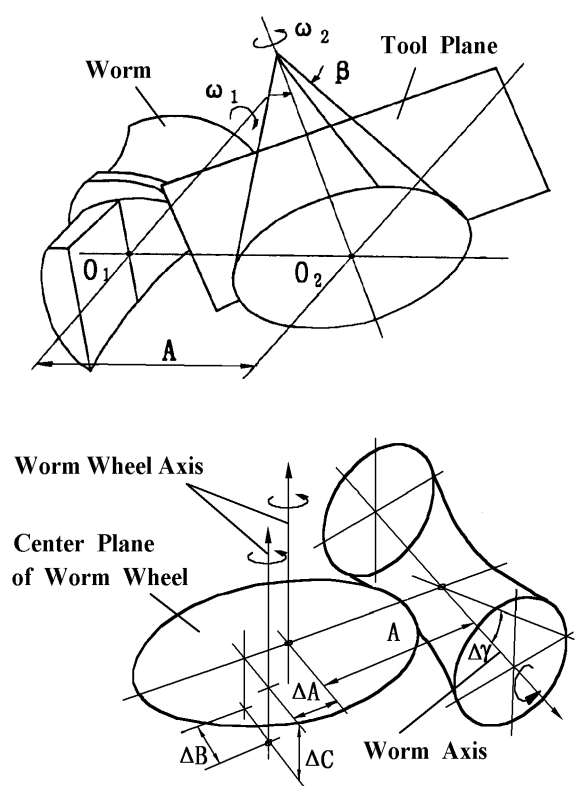

Figure 6 Machining of the worm and assembly

\subsection{Finite element modelling}

The axial profiles of the worm thread at different sections can be obtained from the gear geometry, lofting the profile curves produces the NURBS (Non-Uniform Rational B-Spline) surface of the worm surface. By increasing the amount of the profiles lofted, the NURBS approximation of the worm surfaces can be achieved as precise as desired, then they are merged to create an enclosed volume and to construct the complete solid model of the double enveloping hourglass worm by geometric Boolean operation. Based on the gear geometry of the double enveloping hourglass worm gearing, the tooth surface of the wheel, which is composed of the so-called the original and new contact surfaces, is derived. The original contact surface is a helical plane tooth surface, based on which the solid model can be easily obtained, and the new contact surface is a complicated curved surface formed by so-called new contact lines. By fitting the contact lines the GORDON [9] curved surface of the new contact surface can be obtained. With the amount of the contact lines increases, we get the GORDON approximation of the new contact surface as precise as wanted. From the surfaces obtained and the assembly errors mentioned before, the geometry model of the worm gearing in which the typical errors are included is thus established for finite element analysis. 
Once the tooth surface is known, the surface normal on any point can be determined. A point on the worm surface is specified to locate a node of the contact element. The intersection of the surface normal of the mentioned point with the worm wheel surface identifies another node. In this way all nodes in a zone large enough to cover all of the actual contact elements are defined as the nodes of the contact elements. Each contact element connects a node on the worm surface and its corresponding node on the wheel surface. The constraints on the corresponding displacement components of the relative nodes are imposed.

To minimize the storage and computation time of the model, one third of the complete model is used to make the mesh. All nodes on the rim portion of the wheel are fixed with zero displacement, and the worm is constrained to only rotate about its axis. The mesh model of the worm-wheel assembly is shown in Figure 7.

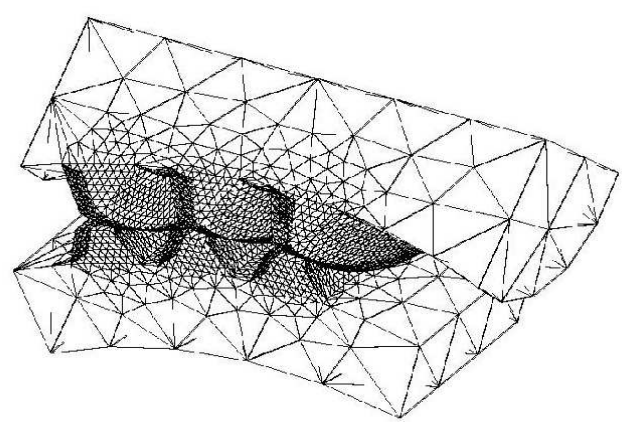

Figure 7. FE model of assembly

\subsection{Loaded tooth contact pattern analysis}

With the finite element models obtained, a loaded tooth contact analysis has been carried out. As shown in Figure 7, the contact FE model contains three contact tooth pairs. Figures 8 and 9 show the contact pattern and pressure distribution on the worm and wheel with the loads $10 \mathrm{kgm}$ and $40 \mathrm{kgm}$ respectively. From the results, it is derived that elastic deformation makes the contact zone spread out from the initial contact lines without load. With increasing load, the contact area and contact pressure increase, but the shape of the contact zone is similar to that without load and is not changed much. From the border to the centre of the contact zone, the contact pressure increases rapidly.

The loaded tooth contact analyses with different errors $\Delta \mathrm{A}$,

$\Delta \mathrm{B}, \Delta \mathrm{C}$ and $\Delta \gamma$ were also conducted, for further details, see [7].

With the finite element analysis results obtained, the following conclusions can be drawn:

- Elastic deformation makes the contact zone spread out from line contact in the case without load. With increasing load, the contact area and contact pressure increase, but the shape of the contact zone are similar to that without load and does not change much. From the border to the center of contact, the contact pressure increases rapidly.

- Load share among the contact tooth pairs of the double enveloping hourglass worm gears is not uniform. From the entrance to the exit of engagement, the load on tooth surface reduces gradually.

- Loaded contact of the double enveloping hourglass worm gears is sensitive to manufacturing errors. When there is manufacturing error, the loaded contact pattern changes usually to the tip or root contact and stress concentration occurs.

- Both load share and contact pattern with manufacturing errors are quite different compared to that without error. When the value of the error is small, with the load increasing, the load share and the contact pattern usually approach the case without error.

- Among the manufacturing errors introduced, the angle error of the crossing axes most significantly influences the contact pattern and the load share. Even if the angle error is small, load increasing could not make the load share and the contact pattern approach the case without error.

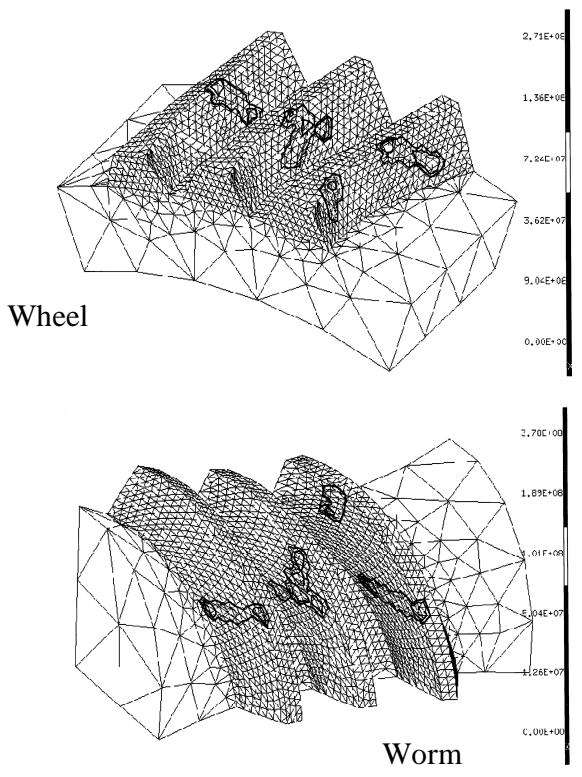

Figure 8. Contact pattern under load $10 \mathrm{kgm}$

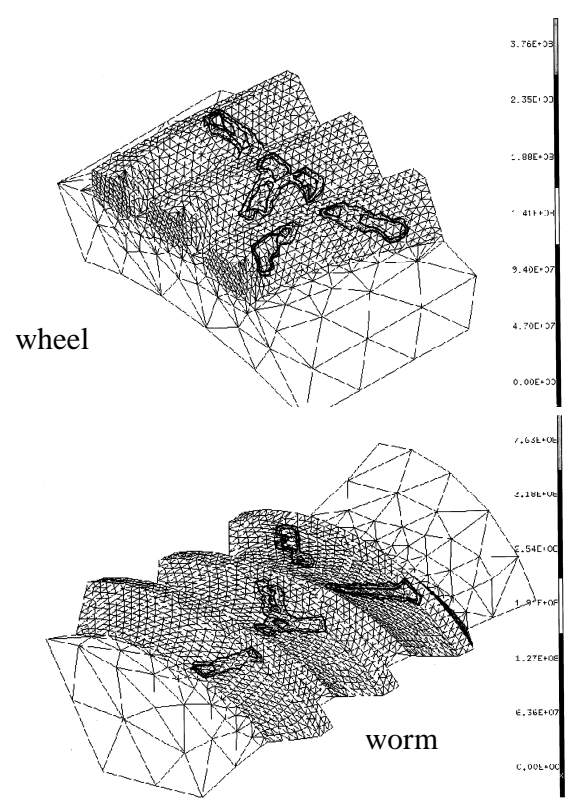

Figure 9 Contact pattern under load $40 \mathrm{kgm}$ 


\section{Concluding remarks}

An integrated approach has been successfully developed for design of worm gear drives. The approach consists of three modules: numerical analysis, three-dimensional-simulation and finite element analysis. It is powerful tool for design of worm gears to achieve high capacity and favourable performance.

Although some methods already exist for worm gear design and the design integration has been seen in some engineering design areas, however, the integration of multiple techniques into the whole design process of worm gearing has not been seen in the literature, and, hence, the approach reported in this paper is an important contribution to worm gear design.

The localised tooth contact for reducing the sensitivities to errors has been widely accepted for worm gearing, however, there is no effective means at the design stage to avoid tooth interference, which often occurs when modifying the tooth geometry to achieve localised tooth contact. As a significant achievement of the new approach, the three-dimensional module ensures that no interference occurs during the tooth mesh and provides a useful visual aid for the designer to investigate mesh process.

The simulation of worm gear hobbing is a novel method to establish the complicated 3-D worm gear model. The model produced by hobbing simulation is parametric and flexible for modification, analysis and optimisation. Also, this approach allows the designer virtually to see the real geometry of the design. Application of this method into gear engineering will bring huge economic benefit.

Loaded tooth contact analysis has been seen important for gear analysis; however, its application in worm gears has not been seen. The finite element analysis module of this approach provides an effective tool to conduct such tasks for worm gears. The results presented in this paper of the loaded tooth contact analysis for both involute and double enveloping worm gear drives are significant achievements.

As presented in this paper, the approach has been applied into the designs of involute and double enveloping worm gear drives. It is a general approach applicable to various types of worm gears. The authors have also applied it into the design of spiroid gear drives [10, 11].

\section{Acknowledgement}

The authors acknowledge the financial supports from the Research Enhancement Funds of The Nottingham Trent University, UK and the Research Grants No. 5977501 and No. 50175112 of the National Science Foundation Council of China (NSFC). Professor Su is grateful for the support from the Cheung Kong Scholars Programme, China.

The authors thank for the following researchers for their contribution to the research reported in this paper: Dr Feng Yang, Dr Yongle Song, Dr Xianfa Wang, Dr Donxing Qin, Dr Yalian Yang and Dr Jianjun Hu.

\section{References}

[1] D Su, F. Yang, R. Gentle, and D Reith, A new approach combining numerical analysis and three-dimensional simulation for design of worm gearing with preferable localized tooth contact, Proceedings of the 1998 ASME Design Engineering Technical Mechanisms Conference, Atlanta, Paper No. DETC98/MECH-5830, 1998

[2] Y. Song and D. Su, Three-dimensional virtual manufacturing of worm gears, Proceedings of the International Conference on Gearing, Transmission and Mechanical Systems, 3-6 July 2000, Nottingham, pp 119128.

[3] F Yang, X. Wang, D Su and C.R. Gentle, A Numerical Method to Obtain Preferable Localised Tooth Contact for Cylindrical Worm Gearing, Proceedings of International Conference on Mechanics in Design, 6th-9th July, Nottingham, UK, 1998, pp 149-157

[4] F. L. Litvin, Gear Geometry and Applied Theory, Prentice Hall, New Jersey, 1994.

[5] F. L. Litvin, J. S. Chen, J. Liu and R. F. Handschun, Application of finite element analysis for determination of load share, real contact ratio, precision of motion, and stress analysis, Journal of Mechanical Design, 118 (4) (1996) 561567.

[6] C. Gosselin, L Cloutier and Q. D. Nguten, A general formulation for the calculation of the load sharing and transmission error under load of spiral bevel and hypoid gears, Mechanism and Machine Theory, 30 (3) (1995) 433450.

[7] Datong Qin and Donxing Qin, Tooth contact analysis of hourglass worm gearing on the condition that errors mixed with deformation, Proceedings of the International conference on gearing, transmission and mechanical systems, 3-6 July 2000, Nottingham, pp 109-118.

[8] D. Su, F. Yang and C. R. Gentle, Analysis of tooth stresses, mesh stiffness and load distribution of involute worm gears, International Journal of Gearing and Transmissions, ISSN 1335-518X, Issue No. 3 (2000) 36-44.

[9] F. Shi, Computer aided geometric design and non-uniform rational B-spline, Beijing Aeronautics and Aerospace University Publishing ltd, Beijing, 1994

[10]D. Su and Y. Song, Numerical modelling of spiroid gears with localized tooth contact, Proceedings of the International Conference on Gearing, Transmission and Mechanical Systems, 3-6 July 2000, Nottingham, pp 75-90.

[11] D Su, Y Song and C R Gentle, Three-dimensional loaded multi-tooth contact analysis of spiroid gears, International Journal of Gearing and Transmission. Issue No.3 (2001) 8390

[12] F Yang, D Su and C R Gentle, 'Finite element modelling and load share analysis for involute worm gears with localised tooth contact', IMechE Proceedings, Part C: Journal of Engineering Sciences, 215 (C7) (2001) 805-816. 